\title{
Hacia una base normativa mexicana en la medición de calidad de vida relacionada con la salud, mediante el Formato Corto 36
}

\author{
Luis D urán-A renas, MD, Ph D, (1) Katia Gallegos-Carrillo, Lic en Com, M en $C$, (1) \\ Guillermo Salinas-Escudero, Lic en Econ, M en Econ de la Salud, ${ }^{(1)}$ \\ Homero Martínez-Salgado, MD, Ph D. ${ }^{(1)}$
}

\begin{abstract}
Durán-Arenas L, Gallegos-Carrillo K, Salinas-Escudero G, Martínez-Salgado H. Hacia una base normativa mexicana en la medición de calidad de vida relacionada con la salud, mediante el Formato Corto 36. Salud Publica Mex 2004;46:306-315. El texto completo en inglés de este artículo está disponible en: http://www.insp.mx/salud/index.html
\end{abstract}

\section{Resumen}

Objetivo. Presentar los resultados de la aplicación del cuestionario de formato corto 36 (SF-36) en dos estados de México (Sonora y 0 axaca), comparando con los niveles de calidad de vida en las provincias de Canadá y en Estados U nidos de América. Material y métodos Los datos provienen de la encuesta de acceso, uso y calidad de los servicios de salud en dos estados de México. El instrumento del SF-36 incluye 36 preguntas que generan ocho dimensiones diferentes sobre calidad de vida relacionada con la salud; a partir de estas dimensiones, se estima un componente físico y otro mental. Para el análisis se calcularon las calificaciones promedio para cada dimensión del SF-36 para toda la muestra, por género y grupos de edad, en cada estado. Las diferencias regionales se evaluaron mediante la diferencia en los promedios. Resultados. Se obtuvo respuesta de $98.5 \%$ de las 4200 viviendas seleccionadas, con porcentajes de respuesta similar en 0 axaca y Sonora. Respondieron al cuestionario 5961 individuos mayores de 25 años de edad. Los hombres tuvieron mayores puntajes que las mujeres en todas las dimensiones y en los dos componentes. En uno u otro sexo la mayor variación en los adultos hasta de 64 años de edad tendió a concentrarse en tres dimensiones: salud general, función social y salud mental; en el grupo de 65 a 74 años de edad se concentró en función social y salud mental; $y$ en mayores de 75 años, en salud mental. Se observó variación en un número mayor de dimensiones en los grupos de adultos jóvenes ( 25 a 44 años), mientras que en

\author{
Durán-Arenas L, Gallegos-Carrillo K, Salinas-Escudero G, \\ Martínez-Salgado $\mathrm{H}$. \\ Towards a Mexican normative standard \\ for measurement of the Short Format 36 \\ health-related quality of life instrument. \\ Salud Publica Mex 2004;46:306-315. \\ The English version of this paper \\ is available at: http://www.insp.mx/salud/index.html
}

Este proyecto fue financiado por el International D evelopment Research Center de Canadá.

(1) Instituto Mexicano del Seguro Social. México, DF, México.

Fecha de recibido: 5 de septiembre de 2003 - Fecha de aprobado: 13 de febrero de 2004

Solicitud de sobretiros: Dr. Luis Durán A renas. Reforma 476, 3er. Piso, colonia Juárez, D elegación C uauhtémoc 06600, México, DF, México.

Correo electrónico: luis.duran@ imss.gob.mx 
las mujeres se observó una mayor variación en los extremos, en los grupos de edad de 25 a 34 años de edad y de 75 y más. Los puntajes promedio en nuestra muestra fueron más altos que los observados en Canadá y Estados U nidos de América en cinco de las dimensiones y en los dos componentes, en tanto que Canadá presento puntajes más altos en las tres dimensiones escalas restantes (salud general, función social y salud mental). Conclusiones En ausencia de datos representativos del ámbito nacional, queremos proponer el uso de nuestros resultados como una base normativa en México, para que los investigadores que utilicen el SF-36 puedan comparar sus resultados con referencias ajustadas por género y edad a escala poblacional. Puesto que las dos regiones estudiadas son claramente contrastantes en su desarrollo social y económico, cuando se trate de pacientes de regiones con mayor desarrollo económico se recomendaría utilizar los datos de Sonora como estándar normativo, y 0 axaca en el caso de pacientes de regiones con menor desarrollo económico. El texto completo en inglés de este artículo está disponible en:http://www.insp.mx/ salud/index.html

Palabras clave:calidad de vida relacionada con la salud; valores normativos; variación regional; México those found in Canada and the United States in five domains and in the two summary components. Canada showed higher scores in the other three domains (overall health, social function, and mental health). Conclusions. SF-36 data are not available at the national level; hence, the authors recommend that their study results may serve as the normative reference for SF-36 in Mexico. Researchers using the SF-36 could compare their results with our reference, adjusted by gender and age in population-based studies. Considering that the study regions have contrasting social and economic characteristics, the data from the more developed state, Sonora, may be used as the normative standard. D ata from 0 axaca would be used as the reference for less-developed states. The English version of this paper is available at: http://www.insp.mx/salud/index.html

Key words: quality of life related to health; normative values; regional variations; M exico
$\mathrm{L}$ a medición de la calidad de vida relacionada con la salud se ha convertido en los últimos años en la medida estándar en ensayos clínicos para realizar mediciones de la efectividad de intervenciones que vayan más allá de las medidas epidemiológicas tradicionales de mortalidad y morbilidad.

Un aspecto central en el desarrollo y utilización de estas medidas es evaluar los niveles de calidad de vida obtenidos en estudios de grupos de pacientes con enfermedades específicas, en comparación con el nivel promedio de estas medidas en la población (país, estado), ajustadas por edad y sexo. Si bien hay países, como Canadá y Estados Unidos de América (EUA), en los cuales se han realizado este tipo de mediciones, hasta donde nuestro conocimiento abarca no existen publicaciones de estas medidas poblacionales en México. ${ }^{1-5}$

Un segundo aspecto de gran importancia es valorar la variabilidad que se puede encontrar por región en las mediciones de calidad de vida relacionadas con la salud (CVRS). Esto es, evaluar si la variación entre regiones dentro de un mismo país requeriría el desarrollo de medidas normativas en regiones o estados.

Con miras a enfrentar estos dos problemas, el principal objetivo del presente artículo se orienta a presentar los resultados de la aplicación del cuestionario del formato corto 36 (SF-36, por sus siglas en inglés) en dos estados de México, contrastantes en su desarrollo socioeconómico: Sonora y Oaxaca. ${ }^{6-10}$ Este cues- tionario se aplicó dentro de una encuesta más amplia orientada a identificar los factores asociados con la utilización y la calidad de los servicios de salud en esos estados. Un segundo objetivo del artículo es presentar un contraste entre los niveles de calidad de vida obtenidos en los estados de Sonora y Oaxaca, comparados con la variación encontrada en las provincias de Canadá y en EUA. El primer análisis permitirá contar con una referencia normativa en México, para que los investigadores que utilicen el SF-36 puedan comparar los resultados que obtengan en estudios específicos de pacientes con normas apropiadas de género y edad poblacional. El segundo permitirá enfrentar la pregunta de si la variabilidad regional en México requiere o no el desarrollo de normas regionales específicas para el país.

\section{Material y métodos}

Los datos provienen de la encuesta de acceso, uso y calidad de los servicios de salud en dos estados de México, la cual fue realizada en 1999-2000. Esta encuesta fue parte de un estudio más amplio que buscaba relacionar el acceso, la utilización y la calidad de los servicios de salud con la implantación de paquetes de ajuste macroeconómico en el país. La iniciativa fue financiada por el Centro de Investigación para el Desarrollo Internacional de Canadá (IDRC MAP Health Project). 
La encuesta se basó en un muestreo polietápico que consideró cuatro etapas y abarcó tanto áreas rurales como urbanas. En la primera etapa se clasificaron los estados del país en tres regiones de acuerdo con su desarrollo socioeconómico. Para cada región se seleccionó, en forma aleatoria simple, un estado. * La segunda etapa incluyó la selección aleatoria de municipios y localidades dentro de los estados para las áreas rurales y la selección aleatoria de áreas geoestadísticas básicas (AGEBS) para la ciudad principal de cada estado. Dentro de la ciudad, las AGEBS fueron listadas y se formaron estratos de acuerdo con criterios socioeconómicos, y se tomó una muestra aleatoria de cada estrato. En estas unidades de muestreo se seleccionaron aleatoriamente un total de 1200 viviendas en zonas rurales y 3000 en zonas urbanas. Tanto en zonas urbanas como rurales se mantuvo una reserva de $10 \%$ para la muestra.

Se elaboraron cuatro instrumentos: el primero incluyó variables de la familia; el segundo, información individual, incluyendo el SF-36; el tercero se orientó en aspectos de la salud materno-infantil, y el cuarto, en la utilización de servicios de salud.

El instrumento del SF-36 incluye 36 preguntas que, una vez calificadas, generan ocho dimensiones diferentes sobre la calidad de vida relacionada con la salud, incluyendo funcionamiento físico, desempeño físico, desempeño emocional, dolor corporal, vitalidad, funcionamiento social, salud mental y percepción general de salud. Todas las dimensiones son calificadas en una escala que varía de 0 a 100, con el valor de 100 que representa el mejor estado posible de salud. A partir de estas dimensiones es posible estimar dos componentes de la calidad de vida relacionada con la salud, uno físico y otro mental. Se utilizó el cuestionario de SF-36 en español desarrollado por el grupo de Ware ${ }^{\ddagger} y$ validado§ en la región de Morelos, México, por el equi-

\footnotetext{
* Se decidió utilizar un solo estado por región dado que los recursos con los que se contaba para la investigación impedían llevar a cabo una encuesta representativa de todo el país. Por esta razón, recurrimos a la práctica establecida del muestreo polietápico. Es importante resaltar que se presentan sólo las regiones Sur (Oaxaca) y Norte (Sonora) pues con los recursos de la investigación sólo en estas dos se completó el levantamiento de las encuestas.

‡ Este cuestionario está disponible en: www.SF-36.org

$\S$ El resultado de este ejercicio de validación permitió certificar que el instrumento funciona bien en poblaciones rurales y urbanas mexicanas. Esto nos dio la certeza de poder utilizar este instrumento en el estudio mayor en los estados de Oaxaca y Sonora. Estos resultados se presentan en un artículo que se encuentra en proceso de revisión en una revista de salud pública de América Latina.
}

po de investigación que elaboró el presente artículo; los datos fueron calificados utilizando la metodología desarrollada por este mismo autor. ${ }^{8}$

\section{Método para las estimaciones normativas del SF-36}

Se estimaron las calificaciones promedio para cada dimensión del SF-36 y se calcularon los datos normativos (promedios, desviaciones estándar, intervalos de confianza de $95 \%$ y porcentajes de piso y techo) para toda la muestra, por género y grupos de edad (con incrementos de 10 años), para cada uno de los estados estudiados. El análisis que aquí se presenta ha sido hecho sólo a escala estatal pues el objetivo de este artículo es generar datos normativos en este ámbito para nuestro país. En un artículo subsecuente se analizarán los factores determinantes de la calidad de vida en estos estados y ahí se procederá a hacer un análisis que considere variaciones dentro de los estratos socioeconómicos que la muestra permite identificar en las entidades federativas.

\section{Método para la comparación de variabilidad regional}

Para evaluar las diferencias regionales se utilizó un análisis de diferencia de promedios. Los resultados se reportan con intervalos de confianza de $95 \%$. Se analizaron en forma separada cada combinación de género, grupo de edad y dimensión del SF-36, dando un total de $2 \times 6 \times 8=96$ análisis. El paquete estadístico que se usó fue el Stata 6. Se realizó estadística descriptiva de cada una de las variables, determinando medidas de tendencia central y dispersión, desviación estándar (DE), intervalos de confianza, para las variables continuas, y de proporciones para las variables nominales y categóricas. De acuerdo con la distribución y tipo de variables se realizaron pruebas paramétricas ( $t$ de Student para muestras independientes) y no paramétricas (Mann Withney) y para las variables nominales y categóricas ji cuadrada o prueba exacta de Fisher.

\section{Resultados}

Los datos fueron recolectados entre junio y septiembre de 1999. Se obtuvo respuesta de $98.5 \%$ de las 4200 viviendas seleccionadas. El porcentaje de respuesta fue similar en Oaxaca y Sonora: $98.75 \%$ y $98.25 \%$, respectivamente. Dentro de estas viviendas, respondieron al cuestionario SF-365961 individuos mayores de 25 años de edad (al menos un individuo por vivienda). Se eli- 
minaron del análisis 366 cuestionarios que correspondían a individuos menores de 25 años.

La edad promedio de los entrevistados que respondieron al SF-36 fue de 46.6 años para mujeres (desviación estándar $(\mathrm{DE})=13.4)$ y 47.9 años para hombres $(\mathrm{DE}=13.8)$. La distribución por edad y sexo fue tres años más joven en el estado de Oaxaca (Sonora, promedio de edad mujeres 48.6 años y hombres 49.6 años, $\mathrm{DE}=13.7$ y 14.2, respectivamente; Oaxaca, promedio de edad mujeres 44.8 años y 46.9 años hombres, $\mathrm{DE}=12.7$ y 13.5 , respectivamente).

\section{Valores normativos del SF-36 en México}

Las calificaciones para las ocho dimensiones y los dos índices de resumen del SF-36 variaron claramente por edad, como se muestra en el cuadro I.

Aunque es posible observar en varias de las dimensiones un efecto de techo (el porcentaje de sujetos que recibió la calificación máxima posible: papel físico y papel emocional, $82.2 \%$ y $82.4 \%$, respectivamente), no se encontró un efecto de piso fuerte (la proporción de individuos que calificaron en el nivel mínimo posible: desempeño físico y desempeño emocional $=6.3 \%$ y $6.0 \%$, respectivamente).

La figura 1 presenta la comparación en el puntaje de las ocho dimensiones y los dos índices de resumen para México, Canadá y EUA. Como puede observarse, México en general presenta puntajes más altos que Canadá y EUA en cinco de las dimensiones y los dos índices de resumen, en tanto que Canadá presenta puntajes más altos en las tres dimensiones restantes (salud general, función social y salud mental). Es importante mencionar que en desempeño físico, dolor corporal y en los dos índices de resumen, la diferencia entre los puntajes promedio es superior a los cinco puntos, y los intervalos de confianza no se sobreponen. Esta diferencia es considerada, clínica y socialmente, significativa. ${ }^{3}$

Los puntajes estandarizados promedio por edad y sexo presentaron un patrón semejante al observado en la población general. En cuanto a la puntuación media general por sexo, se puede observar en la figura 2 que los hombres tuvieron mayores puntajes que las mujeres en todas las dimensiones y en los dos índices; esto es congruente con lo observado en Canadá y en otras poblaciones.

Debemos reconocer que para que los datos normativos sean válidos es necesario que provengan de una muestra representativa de la población de interés. A pesar de que, por razones económicas, la muestra en México se limitó a dos entidades federativas, éstas representan dos extremos de desarrollo social y económico del país.*

Por lo tanto, decidimos estudiarlos para valorar las diferencias regionales, con el fin de poder recomendar estrategias de comparación más efectivas para los estudios sobre grupos específicos de pacientes. A continuación se presenta dicho análisis.

\section{Variabilidad regional}

La variación en tamaño de muestra y en porcentaje de respuesta fue mínima entre los dos estados. También se encontró sólo una ligera diferencia en las distribuciones de edad y sexo, con una estructura poblacional ligeramente más joven en Oaxaca (tres años en general, y con la misma proporción tanto en hombres como en mujeres), y con una proporción mayor de mujeres entre los respondientes de Sonora, en comparación con los de Oaxaca (53.7 y 63.7\%, respectivamente).

El cuadro II presenta el puntaje obtenido en los dos estados para cada dimensión del SF-36 y los dos índices de resumen, así como la diferencia entre los estados con los valores de edad y sexo combinados. Como se observa, las diferencias con los valores de edad y sexo combinados rebasan nuestro estándar (más de cinco puntos para ser clínica y socialmente significativas) en cuatro dimensiones y uno de los componentes. Cuando consideramos solamente a las mujeres, las diferencias se mantienen en valores similares. Sin embargo, consideramos necesario analizar el efecto de la distribución por edad, ya que se podría esperar, como sucedió con los datos normativos canadienses, que existiera un fenómeno de cancelación cruzada de los efectos, esto es, que el efecto de edad y sexo observado en un grupo de edad cancelara el efecto en otro estrato.

En los cuadros III y IV se presentan las diferencias de medias que rebasan los cinco puntos por grupos de

\footnotetext{
* Sonora en el año 2000 tenía 2216969 habitantes, 5.2\% de población indígena, 428987 asegurados en el IMSS, contaba con 42 hospitales, 104.8 camas de hospital por 100000 habitantes, y un PIB per cápita de $\$ 60456$ pesos; mientras que Oaxaca tenía 3438765 habitantes, $45.9 \%$ de población indígena, 205890 asegurados por el IMSS, contaba con 37 hospitales, 47.1 camas de hospital por 100000 habitantes y un PIB per cápita de \$21 457 pesos. Fuente: Instituto Nacional de Estadística, Geografía e Informática (INEGI). Estados Unidos Mexicanos. XII Censo General de Población y Vivienda, 2000. Tabulados Básicos y por Entidad Federativa. Bases de Datos y Tabulados de la Muestra Censal. Aguascalientes, Aguascalientes, México, 2001; Sistema de Cuentas Nacionales, INEGI, 2001.
} 


\section{Cuadro I \\ Resultados de las dimensiones y de los índices de Resumen de La SF-36, por GRUPO de edad. Oaxaca y Sonora. México, 1999-2000}

\begin{tabular}{|c|c|c|c|c|c|c|c|c|c|c|}
\hline $\begin{array}{l}\text { Grupo } \\
\text { de edad }\end{array}$ & $\begin{array}{l}\text { Función } \\
\text { física }\end{array}$ & $\begin{array}{l}\text { Rol } \\
\text { físico }\end{array}$ & $\begin{array}{l}\text { Dolor } \\
\text { corporal }\end{array}$ & $\begin{array}{l}\text { Percepción } \\
\text { de salud } \\
\text { general }\end{array}$ & Vitalidad & $\begin{array}{c}\text { Función } \\
\text { social }\end{array}$ & $\begin{array}{c}\text { Rol } \\
\text { emocional }\end{array}$ & $\begin{array}{l}\text { Salud } \\
\text { mental }\end{array}$ & $\begin{array}{l}\text { Componente } \\
\text { de escala } \\
\text { física }\end{array}$ & $\begin{array}{c}\text { Componente } \\
\text { de escala } \\
\text { mental }\end{array}$ \\
\hline $25-34$ & 1175 & 1170 & 1167 & 1175 & 1174 & 1174 & 1174 & 1174 & 1175 & 1175 \\
\hline Valor medio & 96.3 & 93.5 & 89.4 & 54.9 & 74.1 & 77.1 & 93.2 & 74.1 & 83.5 & 79.6 \\
\hline Desviación estándar & 0.3 & 0.6 & 0.6 & 0.4 & 0.5 & 0.5 & 0.6 & 0.5 & 0.3 & 0.4 \\
\hline Intervalo de confianza de $95 \%$ & 95.696 .9 & 92.394 .6 & 88.190 .6 & 54.255 .6 & 73.075 .0 & 76.278 .0 & 92.094 .2 & 73.075 .1 & 82.884 .1 & 78.880 .3 \\
\hline$\%$ al piso & 0.3 & 2.1 & 0.7 & 0.0 & 0.0 & 0.1 & 2.0 & 0.0 & 0.0 & 0.0 \\
\hline$\%$ al techo & 82.6 & 87.0 & 77.2 & 0.0 & 11.8 & 0.3 & 86.0 & 12.3 & 0.0 & 0.2 \\
\hline $35-44$ & 1535 & 1528 & 1533 & 1535 & 1535 & 1534 & 1533 & 1535 & 1535 & 1535 \\
\hline Valor medio & 96.3 & 96.3 & 96.3 & 96.3 & 96.3 & 96.3 & 96.3 & 96.3 & 96.3 & 96.3 \\
\hline Desviación estándar & 0.3 & 0.3 & 0.3 & 0.3 & 0.3 & 0.3 & 0.3 & 0.3 & 0.3 & 0.3 \\
\hline Intervalo de confianza de $95 \%$ & 95.696 .9 & 95.696 .9 & 95.696 .9 & 95.696 .9 & 95.696 .9 & 95.696 .9 & 95.696 .9 & 95.696 .9 & 95.696 .9 & 95.696 .9 \\
\hline$\%$ al piso & 0.3 & 0.3 & 0.3 & 0.3 & 0.3 & 0.3 & 0.3 & 0.3 & 0.3 & 0.3 \\
\hline$\%$ al techo & 82.6 & 82.6 & 82.6 & 82.6 & 82.6 & 82.6 & 82.6 & 82.6 & 82.6 & 82.6 \\
\hline$\underline{45-54}$ & 1566 & 1553 & 1560 & 1568 & 1563 & 1562 & 1563 & 1563 & 1566 & 1584 \\
\hline Valor medio & 96.3 & 96.3 & 96.3 & 96.3 & 96.3 & 96.3 & 96.3 & 96.3 & 96.3 & 96.3 \\
\hline Desviación estándar & 0.3 & 0.3 & 0.3 & 0.3 & 0.3 & 0.3 & 0.3 & 0.3 & 0.3 & 0.3 \\
\hline Inter valo de confianza de $95 \%$ & 95.696 .9 & 95.696 .9 & 95.696 .9 & 95.696 .9 & 95.696 .9 & 95.696 .9 & 95.696 .9 & 95.696 .9 & 95.696 .9 & 95.696 .9 \\
\hline$\%$ al piso & 0.3 & 0.3 & 0.3 & 0.3 & 0.3 & 0.3 & 0.3 & 0.3 & 0.3 & 0.3 \\
\hline$\%$ al techo & 82.6 & 82.6 & 82.6 & 82.6 & 82.6 & 82.6 & 82.6 & 82.6 & 82.6 & 82.6 \\
\hline $55-64$ & 978 & 973 & 975 & 978 & 976 & 976 & 976 & 976 & 978 & 976 \\
\hline Valor medio & 84.9 & 84.7 & 82.2 & 49.9 & 69.1 & 74.5 & 86.6 & 71.2 & 75.4 & 75.3 \\
\hline Desviación estándar & 0.7 & 1.0 & 0.8 & 0.4 & 0.6 & 0.6 & 1.0 & 0.6 & 0.6 & 0.5 \\
\hline Intervalo de confianza de $95 \%$ & 83.486 .3 & 82.686 .6 & 80.583 .7 & 49.150 .7 & 68.070 .1 & 73.375 .5 & 84.788 .5 & 70.072 .2 & 74.376 .5 & 74.376 .3 \\
\hline$\%$ al piso & 0.7 & 9.0 & 1.1 & 0.1 & 0.0 & 0.3 & 8.2 & 0.0 & 0.0 & 0.0 \\
\hline$\%$ al techo & 52.5 & 77.7 & 59.4 & 0.1 & 6.6 & 0.1 & 80.1 & 7.0 & 0.1 & 0.0 \\
\hline $65-74$ & 483 & 481 & 481 & 483 & 481 & 481 & 483 & 481 & 483 & 483 \\
\hline Valor medio & 70.2 & 72.1 & 73.1 & 47.6 & 65.3 & 69.9 & 74.8 & 70.3 & 65.8 & 70.1 \\
\hline Desviación estándar & 1.3 & 1.8 & 1.4 & 0.6 & 0.9 & 0.9 & 1.8 & 0.9 & 1.0 & 0.9 \\
\hline Intervalo de confianza de $95 \%$ & 67.672 .9 & 68.575 .7 & 70.375 .8 & 46.448 .7 & 63.566 .9 & 68.171 .7 & 71.378 .3 & 68.572 .0 & 63.867 .7 & 68.471 .8 \\
\hline$\%$ al piso & 3.7 & 18.5 & 2.7 & 0.2 & 0.0 & 0.6 & 17.8 & 0.0 & 0.0 & 0.0 \\
\hline$\%$ al techo & 30.8 & 62.1 & 46.8 & 100.0 & 4.6 & 0.2 & 66.9 & 6.7 & 0.2 & 0.2 \\
\hline $75+$ & 204 & 203 & 204 & 204 & 204 & 204 & 202 & 204 & 204 & 204 \\
\hline Valor medio & 56.0 & 58.5 & 69.0 & 45.4 & 59.4 & 62.6 & 61.1 & 66.3 & 57.2 & 62.4 \\
\hline Desviación estándar & 2.1 & 3.1 & 2.1 & 1.0 & 1.4 & 1.5 & 3.1 & 1.4 & 1.6 & 1.4 \\
\hline Intervalo de confianza de $95 \%$ & 51.860 .1 & 52.464 .6 & 64.873 .1 & 43.447 .3 & 56.662 .2 & 59.765 .4 & 55.067 .2 & 63.669 .0 & 54.160 .3 & 59.565 .2 \\
\hline$\%$ al piso & 4.9 & 30.1 & 1.5 & 0.0 & 0.5 & 1.0 & 29.2 & 0.0 & 0.0 & 0.0 \\
\hline$\%$ al techo & 14.7 & 46.8 & 38.2 & 0.0 & 2.0 & 0.0 & 50.0 & 4.9 & 0.0 & 0.0 \\
\hline Todas las edades & 5950 & 5916 & 5928 & 5950 & 5941 & 5939 & 5939 & 5941 & 5950 & 5945 \\
\hline Valor medio & 89.6 & 88.7 & 85.5 & 52.2 & 70.7 & 75.1 & 88.9 & 72.1 & 79.0 & 76.7 \\
\hline Desviación estándar & 0.3 & 0.4 & 0.3 & 0.2 & 0.2 & 0.2 & 0.3 & 0.2 & 0.2 & 0.2 \\
\hline Intervalo de confianza de $95 \%$ & 89.090 .1 & 87.989 .3 & 84.986 .1 & 51.852 .5 & 70.271 .1 & 74.775 .5 & 88.289 .6 & 71.572 .5 & 78.579 .3 & 76.377 .1 \\
\hline$\%$ al piso & 0.8 & 6.3 & 0.9 & 0.0 & 0.1 & 0.3 & 6.0 & 0.0 & 0.0 & 0.0 \\
\hline$\%$ al techo & 67.1 & 82.2 & 68.2 & 0.1 & 9.0 & 0.3 & 82.4 & 10.0 & 0.0 & 0.1 \\
\hline
\end{tabular}

El \% de piso y techo se refiere a la proporción de encuestados con la puntuación mínima (0) y máxima (100) posible, respectivamente 


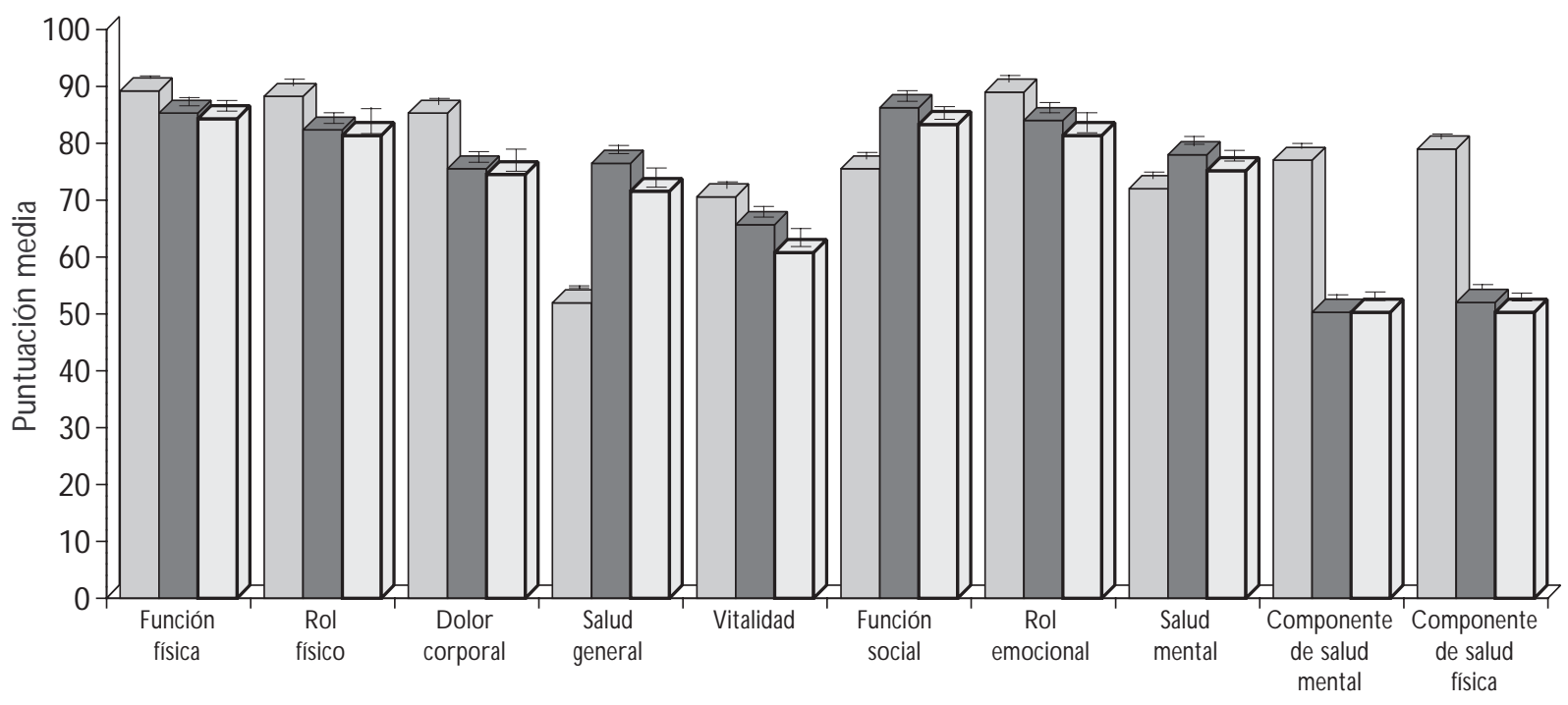

Dimensiones de calidad de vida del SF-36

México

Canadá

Estados Unidos de América

Figura 1. Puntaje de las ocho dimensiones del SF-36 y los componentes de salud física y mental. México, Canadá y Estados Unidos de América

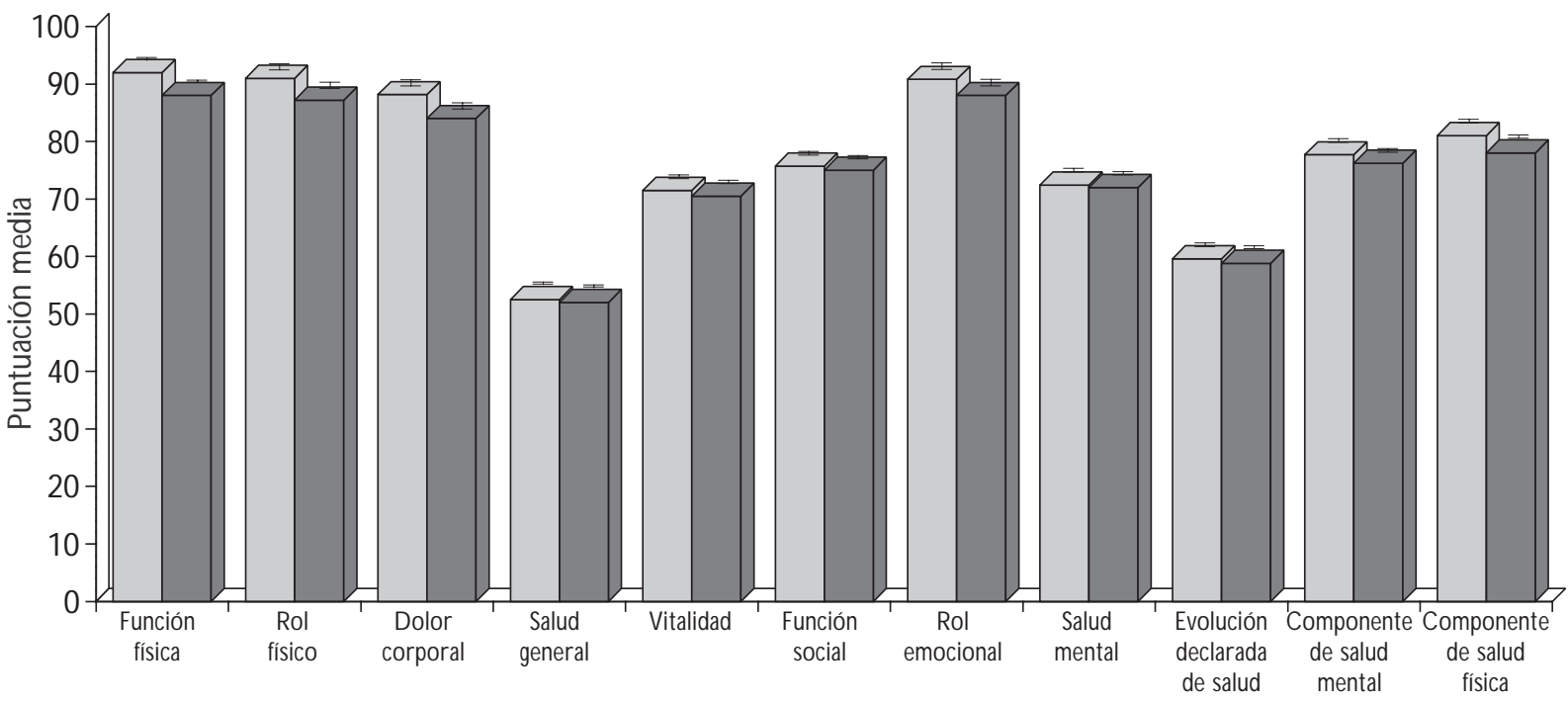

Dimensiones de calidad de vida del SF-36

Hombres

Mujeres

Figura 2. Puntuación media general de las dimensiones, los índices de Resumen y la evolución declarada de salud, del instrumento SF-36 para México, por sexo. Oaxaca y Sonora. México, 1999-2000 


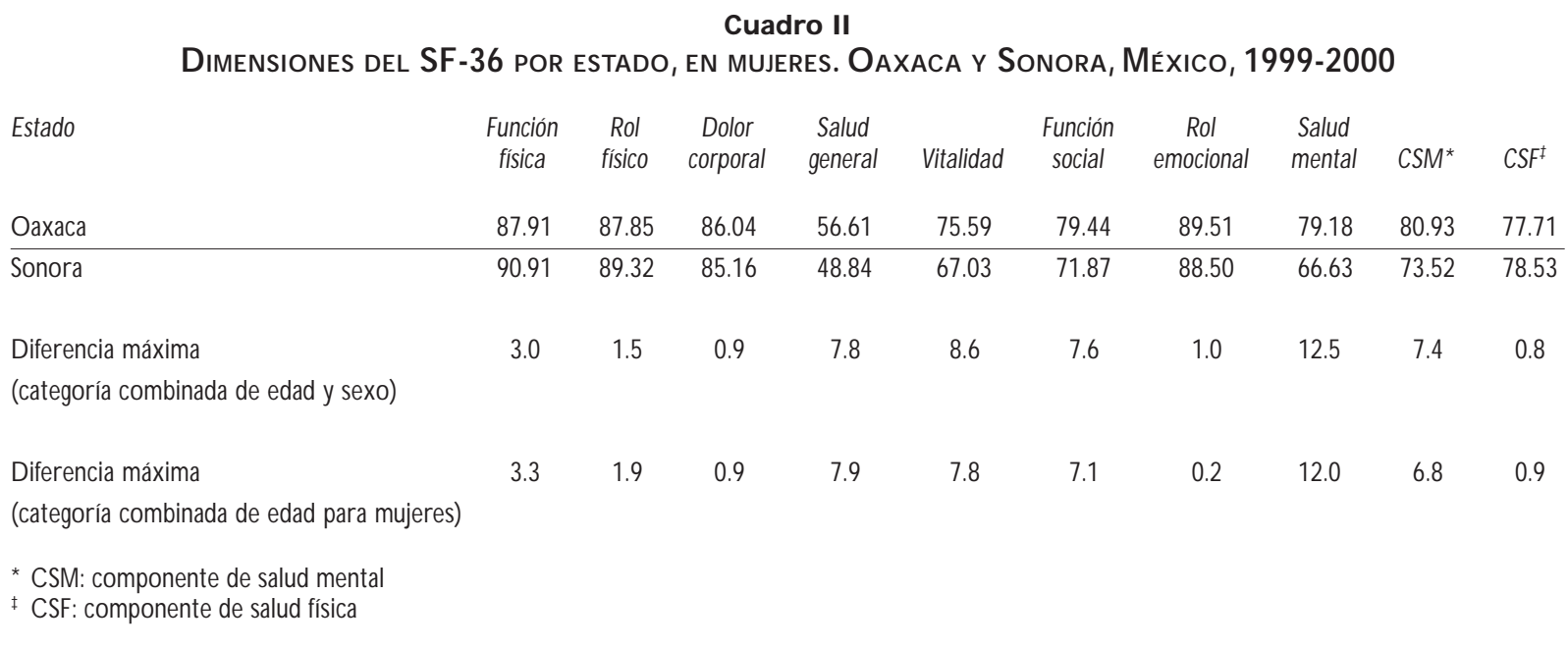

edad y sexo. Se puede observar que existe una gran variabilidad, tanto en hombres como en mujeres y en múltiples dimensiones. Llama la atención que, tanto en hombres como en mujeres, la mayor variación que se observa en los adultos hasta de 64 años de edad tiende a concentrarse en tres dimensiones: salud general, función social y salud mental; en el grupo de 65 a 74 años de edad se concentra en función social y salud mental; y en mayores de 75 años de edad, en salud mental.

Es importante resaltar que existe un patrón inverso entre hombres y mujeres en el número de dimensiones en las que encontramos variaciones clínica y socialmente significativas. Así, se observa variación en un número mayor de dimensiones en los grupos de adultos jóvenes ( 25 a 44 años de edad), mientras que en las mujeres se observa una mayor variación en los extremos, en los grupos de edad de 25 a 34 años y de 75 años y más.

\section{Discusión}

La necesidad de tener datos normativos de países específicos para comparar el efecto sobre la calidad de vida relacionada con la salud en grupos de pacientes específicos está sustentada en los hallazgos en Canadá, EUA y, ahora, en México.

En contraste con lo encontrado en otros países, en México parecen existir importantes variaciones regionales, como lo sugieren nuestros resultados en el caso de Sonora y Oaxaca. En efecto, a pesar de que la muestra de estudio se restringió a dos regiones, claramente contrastantes en su desarrollo social y económico, esto nos da pie para discutir varios aspectos. Aunque den- tro de estos extremos se encuentra la realidad del país, no podemos aceptar que el promedio de estos extremos refleje el promedio real del país. Primero, sería necesaria la medición de calidad de vida relacionada con la salud en una encuesta representativa nacional. Esto nos permitiría generar datos normativos de calidad de vida relacionada con la salud que resultaran más adecuados para hacer comparaciones generales. En segundo lugar, mientras se logra lo anterior, quisiéramos proponer el uso de los dos extremos que hemos presentado en este artículo como datos normativos, para ser utilizados de acuerdo al origen de los grupos de pacientes con los cuales se quiere comparar: si son pacientes de regiones con mayor desarrollo económico, se recomendaría utilizar los datos de Sonora como estándar normativo, en tanto que en el caso de pacientes de regiones con menor desarrollo económico proponemos usar los datos de Oaxaca.

Aceptando la propuesta de que los datos presentados pueden ser tomados como normativos para México, llama la atención que, en general, los puntajes promedios son más altos en México que en Canadá y EUA. Esto puede deberse, en parte, a que el promedio de edad de la población que respondió al SF-36 en México fue aproximadamente 14 años menor que la población canadiense y la estadounidense. Al mismo tiempo, este hallazgo es consistente con la variabilidad regional que se ha encontrado en otros estudios, y señala la necesidad de utilizar datos normativos por país para las comparaciones de efectos de estudios en grupos de pacientes en países específicos.

Sin embargo, no hay que perder de vista que las diferencias entre países pueden también deberse a diferencias metodológicas entre los estudios. En el caso 


\section{Cuadro III \\ DiFERENCIA DE MEDIAS POR GRUPOS DE EDAD \\ Y ESTADO, EN HOMBRES. OAXACA \\ y Sonora, México, 1999-2000}

\begin{tabular}{|c|c|c|c|c|}
\hline \multirow{3}{*}{$\begin{array}{l}\text { Escala } \\
\text { Rol físico }\end{array}$} & \multirow[b]{2}{*}{ Grupo de edad } & \multirow{2}{*}{$\begin{array}{c}\text { Diferencia } \\
\text { (Intervalo de confianza) }\end{array}$} & \multicolumn{2}{|c|}{ Media por estado } \\
\hline & & & 0axaca & Sonora \\
\hline & $25-34$ & 6.81 & 92.42 & 99.23 \\
\hline & \multicolumn{4}{|c|}{ (3.52 10.101) } \\
\hline \multirow{2}{*}{ Dolor corporal } & $25-34$ & 7.06 & 88.58 & 95.64 \\
\hline & \multicolumn{4}{|c|}{ (3.23 10.896) } \\
\hline \multirow[t]{2}{*}{ Salud general } & $25-34$ & 11.59 & 51.30 & 62.90 \\
\hline & \multicolumn{4}{|c|}{ (9.56 13.662) } \\
\hline \multirow[t]{2}{*}{ Vitalidad } & $25-34$ & 15.35 & 69.66 & 85.01 \\
\hline & \multicolumn{4}{|c|}{ (12.411 18.284) } \\
\hline \multirow[t]{2}{*}{ Función social } & $25-34$ & 10.15 & 73.63 & 83.78 \\
\hline & \multicolumn{4}{|c|}{ (7.299 12.994) } \\
\hline \multirow[t]{2}{*}{ Rol emocional } & $25-34$ & 3.42 & 92.25 & 95.67 \\
\hline & \multicolumn{4}{|c|}{$3.49 .432)$} \\
\hline \multirow[t]{2}{*}{ Salud mental } & $25-34$ & 16.72 & 68.84 & 85.56 \\
\hline & \multicolumn{4}{|c|}{ (13.525 19.924) } \\
\hline \multirow[t]{2}{*}{ Salud general } & $35-44$ & 10.94 & 50.26 & 61.20 \\
\hline & \multicolumn{4}{|c|}{ (9.2 12.668) } \\
\hline \multirow[t]{2}{*}{ Vitalidad } & $35-44$ & 11.72 & 68.53 & 80.25 \\
\hline & \multicolumn{4}{|c|}{ (8.951 14.488) } \\
\hline Función social & $35-44$ & 10.03 & 73.34 & 83.37 \\
\hline & & (7.589 12.46) & & \\
\hline Rol emocional & $35-44$ & 5.68 & 91.83 & 97.51 \\
\hline & & $(2.4958 .866)$ & & \\
\hline Salud mental & $35-44$ & 14.81 & 67.63 & 82.43 \\
\hline & & (11.937 17.676) & & \\
\hline Salud general & $45-54$ & 10.39 & 49.34 & 59.73 \\
\hline & & (8.557 12. 227) & & \\
\hline Vitalidad & $45-54$ & 12.32 & 67.70 & 80.01 \\
\hline & & (9.73 14.898) & & \\
\hline Función social & $45-54$ & 10.59 & 72.26 & 82.85 \\
\hline & & (8.144 13.039) & & \\
\hline Salud mental & $45-54$ & 16.00 & 66.55 & 82.55 \\
\hline & & (13.362 18.631) & & \\
\hline Función física & $55-64$ & 6.29 & 91.46 & 85.16 \\
\hline & & (2.455 10.129) & & \\
\hline $\begin{array}{l}\text { Vitalidad } \\
\end{array}$ & $55-64$ & 7.16 & 67.10 & 74.26 \\
\hline & & (3.899 10.43) & & \\
\hline Función social & $55-64$ & 7.26 & 72.56 & 79.82 \\
\hline & & (4.247 10.275) & & \\
\hline Salud mental & $55-64$ & 12.15 & 66.77 & 78.92 \\
\hline & & (8.892 15.412) & & \\
\hline Vitalidad & $65-74$ & 6.14 & 64.32 & 70.46 \\
\hline & & (1.564 10.719) & & \\
\hline Función social & $65-74$ & 4.99 & 70.26 & 75.25 \\
\hline & & $(0.2339 .754)$ & & \\
\hline Salud mental & $65-74$ & 9.02 & 67.42 & 76.44 \\
\hline & & (4.52 13.525) & & \\
\hline Función social & $75+$ & 9.17 & 60.59 & 69.76 \\
\hline & & $(0.807$ 17.539) & & \\
\hline Salud mental & $75+$ & 12.02 & 61.10 & 73.11 \\
\hline
\end{tabular}

\section{Cuadro IV \\ Diferencia De MEDIAS POR GRUPOS DE EDAD \\ Y ESTADO, EN MUJERES. OAXACA \\ y Sonora, México, 1999-2000}

Diferencia Media por estado Grupo de edad (Intervalo de confianza) Oaxaca Sonora

$\begin{array}{lrrrr}\text { Dolor corporal } \quad 25-34 & 5.78 & 86.00 & 91.77\end{array}$ (2.277 9.274)

\begin{tabular}{lllll}
\hline Salud general & $25-34$ & 12.58 & 49.78 & 62.36
\end{tabular}

\begin{tabular}{lcccc}
\hline \multicolumn{5}{c}{$(10.91514 .24)$} \\
\hline Vitalidad & $25-34$ & 10.15 & 69.46 & 79.62 \\
\hline
\end{tabular}

\begin{tabular}{lcccc}
\hline & $(7.70612 .598)$ \\
\hline Función social & $25-34$ & 9.77 & 73.27 & 83.04
\end{tabular}

\begin{tabular}{lcccc}
\hline \multicolumn{5}{c}{$(7.45812 .08)$} \\
\hline Salud mental & $25-34$ & 13.09 & 68.57 & 81.66
\end{tabular}

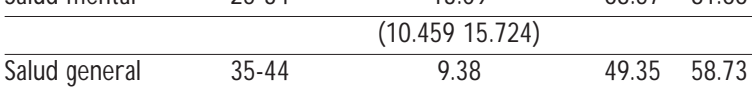

\begin{tabular}{lcccc}
\hline Salud general & $35-44$ & 9.38 & 49.35 & 58.73 \\
\hline & & $(7.94910 .818)$ & &
\end{tabular}

\begin{tabular}{lcccc}
\hline Vitalidad & $35-44$ & 10.32 & 66.05 & 76.37
\end{tabular}

\begin{tabular}{lcccc}
\hline \multicolumn{5}{c}{$(8.05312 .577)$} \\
\hline Función social & $35-44$ & 9.25 & 72.26 & 81.51 \\
\hline
\end{tabular}

\begin{tabular}{lcccc}
\hline \multicolumn{5}{c}{$(7.23211 .258)$} \\
\hline Salud mental & $35-44$ & 13.98 & 65.85 & 79.83 \\
\hline
\end{tabular}

\begin{tabular}{|c|c|}
\hline & \multirow{2}{*}{$\begin{array}{c}(11.66816 .286) \\
9.10\end{array}$} \\
\hline lud gen & \\
\hline
\end{tabular}

\begin{tabular}{lcccc}
\hline \multicolumn{5}{c}{$(7.5710 .61)$} \\
\hline Vitalidad & $45-54$ & 10.71 & 66.46 & 77.16 \\
\hline
\end{tabular}

\begin{tabular}{|c|c|c|c|c|}
\hline & & 7912 & & \\
\hline Función social & $45-54$ & 7.94 & 72.10 & 80.04 \\
\hline & & 36210 & & \\
\hline Salud mental & $45-54$ & 14.60 & 65.21 & 79.82 \\
\hline & & 3571 & & \\
\hline Rol físico & 55-64 & 5.62 & 84.16 & 78.54 \\
\hline & & 2711 & & \\
\hline Salud general & $55-64$ & 4.62 & 47.16 & 51.78 \\
\hline & & 4246. & & \\
\hline Función social & $55-64$ & 6.24 & 70.37 & 76.61 \\
\hline & & 1619. & & \\
\hline Salud mental & $55-64$ & 7.65 & 66.84 & 74.49 \\
\hline
\end{tabular}

\begin{tabular}{lcccc}
\hline Salud mental & $55-64$ & 7.65 & 66.84 & 74.49 \\
\hline & \multicolumn{4}{c}{$(4.63510 .657)$} \\
\hline Dolor corporal & $65-74$ & 6.21 & 66.70 & 72.91 \\
\hline
\end{tabular}

\begin{tabular}{lcccc}
\hline Dolor corporal & $65-74$ & 6.21 & 66.70 & 72.91 \\
\hline & \multicolumn{4}{c}{$(1.74214 .166)$} \\
\hline Función social & $65-74$ & 5.68 & 63.66 & 69.34 \\
\hline
\end{tabular}

\begin{tabular}{lcccc}
\hline Función social & $65-74$ & 5.68 & 63.66 & 69.34 \\
\hline \multicolumn{5}{c}{$(0.4710 .893)$} \\
\hline Salud mental & $65-74$ & 10.45 & 62.16 & 72.61 \\
\hline & \multicolumn{5}{c}{$(5.3115 .585)$} \\
\hline Función física & $75+$ & 12.89 & 59.72 & 46.83
\end{tabular}

\begin{tabular}{lcccc}
\hline Función física & $75+$ & 12.89 & 59.72 & 46.83 \\
\hline \multicolumn{5}{c}{$(1.43624 .334)$} \\
\hline Rol físico & $75+$ & 27.09 & 73.41 & 46.32 \\
\hline
\end{tabular}

\begin{tabular}{lcccc}
\hline Rol físico & $75+$ & 27.09 & 73.41 & 46.32 \\
\hline & \multicolumn{4}{c}{$(10.50243 .657)$} \\
\hline Dolor corporal & $75+$ & 14.07 & 74.65 & 60.58 \\
\hline
\end{tabular}

\begin{tabular}{lcccc}
\hline Dolor corporal & $75+$ & 14.07 & 74.65 & 60.58 \\
\hline & \multicolumn{5}{c}{$(2.201125 .924)$} \\
\hline Rol emocional & $75+$ & 16.90 & 70.63 & 53.73 \\
\hline \multicolumn{5}{c}{$(0.34934 .156)$} \\
\hline Salud mental & $75+$ & 10.36 & 59.81 & 70.17 \\
\hline
\end{tabular}

(3.081 17.641) 
de Canadá, se trató de poblaciones localizadas en un radio de $50 \mathrm{~km}$ de distancia alrededor de nueve ciudades importantes, en tanto que en EUA se basó en la respuesta de 2474 participantes en la encuesta nacional de estado funcional, que fueron seleccionados en forma no aleatoria entre los participantes de una encuesta previa (la encuesta social general). En el caso de México se trató de una encuesta de zonas urbanas y rurales. Estas diferencias resaltan la importancia de señalar que resulta esencial contar con la descripción metodológica del estudio de donde se derivan los datos normativos, con el fin de poder hacer comparaciones válidas.

Las diferencias que se encontraron entre los puntajes por sexo en la muestra de los estados de Sonora y Oaxaca son consistentes con los encontrados en otros países como el Reino Unido, Canadá y EUA, ${ }^{3,4,11,12}$ aunque en general las diferencias no rebasaban en ningún caso los cinco puntos mínimos señalados por el consenso internacional para considerar las diferencias significativas clínica y socialmente.

Sin duda, las medidas de calidad de vida relacionada con la salud llegaron para quedarse, y seguramente experimentarán un crecimiento explosivo dada la composición de nuestra población, la cual, en un lapso no mayor de 25 años, alcanzará la situación que se observa en países europeos. En consecuencia, nuestra última reflexión se centra en recomendar la necesidad de apoyar este tipo de estudios, tanto para el SF-36 como para el Euroqol, otra medida de CVRS cada vez más utilizada en estudios de evaluación de tecnología, ensayos clínicos aleatorios y estudios de costo-efectividad. ${ }^{13-29}$ Cuando estas medidas se apliquen a grupos de pacientes específicos, será necesario contar con datos normativos nacionales e inclusive regionales para valorar correctamente el efecto de las intervenciones (por ejemplo, medicamentos); en ausencia de estos datos normativos no será posible valorar verdaderamente las contribuciones de las intervenciones medicamentosas, de manera que sea posible hacer comparaciones, manteniendo constante el efecto de otros padecimientos o factores observables en la población.

\section{Referencias}

1. Zúñiga AM, C arrillo JG T, Fos PJ, G andek B, Medina MRM. Evaluación del estado de salud con la encuesta SF-36. Resultados preliminares en México. Salud Publica Mex 1999; 41: 110-118.

2.Velarde E,Avila C. Evaluación de la calidad de vida. Salud Publica Mex; 44(4):349-361.
3. Hopman W M, Towheed T,A nastassiades T, Tenenhouse A, Poliquin S, Berger $\mathrm{C}$ et al. $\mathrm{C}$ anadian normative data for the Sf-36:The need for regional norms.J Public Health Med 1995: 17: 46-50.

4. Hopman W M, Berger C, Joseph L, Towheed T,A nastassiades T, Tenenhouse A et al. Is there regional variation in the SF-36 scores of Canadian adults? C an J Public Health. 2002 May-Jun;93(3):233-237. 5. W ood-D auphinne SW. The Canadian SF-36 health survey. N ormative data add to its value. CMAJ 2000;163:284-284.

6. W are JE Jr, Kosinski M, Bayliss MS, MCH orney CA, Rogers W H, Raczek A. Comparison of methods for the scoring and statistical analysis of SF36 health profile and measures: Summary of results from the Medical 0 utcomes Study. Med Care 1995;33 (4 suppl):AS264-AS279.

7. W are JE Jr, Sherbourne CD.The MOS 36-item short form health survey (SF-36): I. Conceptual framework and item selection. Med Care 1992; 30:473-483.

8.W are JE. SF-36 health survey manual and interpretation guide. Boston (MA): The Health Institute, $\mathrm{N}$ ew England Medical Centre; 1993.

9.W are JE.The SF-36 health survey. En: Spilker B, ed. Q uality of life and pharmaco-economics in clinical trials. Second Edition. Philadelphia (PA): Lippincott-Raven Publisher; 1996:337-345.

10. W are JE, Kosinski M, Keller SD. SF-36 physical and mental health summary scale:A U ser's manual. Boston (MA):The Health Institute, $\mathrm{N}$ ew England Medical Centre; 1994.

11. Jenkinson C, Stewart-Brown S, Petersen S, Paice C. Assessment of the SF-36 version 2 in the United Kingdom.J Epidemiol Community Health 1999; 53:46-50.

12. Jenkinson $C$, C oulter $C, W$ right L. Short form 36 (SF-36) health survey questionnaire: $\mathrm{N}$ ormative data for adults of working age. BM] 1993;306:1437-1440.

13. Beaton DE, Hogg-Johnson S, Bombardier C. Evaluating changes in health status: Reliability and responsiveness of five generic health status measures in workers with musculoskeletal disorders. J C lin Epidemiol 1997;50: 79-93.

14. Berzon RA, Understanding and using health- related quality of life instruments within clinical research studies. En: Staquet MJ, H ays RD,

Fayers FM, ed. Q uality of life assessments in clinical trials: Methods and practice. 0 xford: 0 xford University Press; 1998:3-15.

15. Essink-Bot ML, Krabbe PFM, Bonsel GJ,Aaroson N K.An empirical comparison of four generic health status measures. Med Care 1997;35:522-537.

16. Hemingway H, Stafford M, Stanfield S, Shipley M, Marmot M. Is the SF-36 a valid measure of change in population health? Result from the W hitehall II study. BMJ 1997; 315:1273-1279.

17. Kreiger N, Tenenhouse A, Joseoh L, Mackenzie T, Poliquin S, Brown JP et al.The $\mathrm{C}$ anadian Multicentre 0 steoporosis Study (Camos): Background, rationale, methods. C an J Aging 1999;18: 376-387.

18. Lyons RA, Fielder $\mathrm{H}$, Littlepage NC. Measuring health status with the SF-36:The need for regional norms.J Public Health Med 1995; 17:46-50. 19. McHorney CA, W are JE Jr, Raczek AE.Them MOS 36-item short form health survey (SF-36): II. Psychometric and clinical test of validity in measuring physical and mental health constructs. Med Care 1993;31: 247-263.

20. McH orney CA,W are JE Jr,Lu JFR, Sherbourne CD.The MOS 36-item short form health survey: Ill. Test of data quality, scaling assumptions, and reliability across diverse patient groups. Med Care 1994;32: 40-66. 21. Melzoff J. Critical thinking about research: Psychology and related fields. W ashington, DC:A merican Psychological Associations; 1998. 22. Mishra G, Schofield MJ. N orms for the physical and mental health component summary scores of the SF-36 for young, middle-aged and older Australian women. Q ual Life Res 1998; 7:215-220.

23. Raftery $A$, Lewis $S$. How many iterations in the G ibbs sampler? En: Bernardo JM, Berger JO , D awid JO, Smith AFM, ed. Bayesian statistics 4. 0 xford: O xford University Press, 1992;763-773. 
24. Rubin D. Multiple imputation for non-respondents in surveys. N ew York (N Y): John W iley \& Sons; 1987.

25. Spiegelhter DJ,Thomas A, Best N G, G ilks W R. BU GS: Bayesian inference using $\mathrm{G}$ ibbs sampling,Version 0.5, (version ii). C ambridge, UK: MRC Biostatistics Unit; 1996.

26. Statistics $C$ anada provincial census data, 1991. Age, sex and marital status. The N ation 0 ttawa: Statistics C anada; 1993. (Cat. N 0. 93-310). 27. Statistics C anada Provincial Census Data, 1991. Profile of census metropolitan areas and census agglomeration, part A. 0 ttawa: Statistics Canada; 1993. (C at. N 0. 93-337).
28.W atson EK, Firman DW, Baade PD, Ring I.Telephone administration of the SF-36 health survey:Validation studies and population norms for adults in Q ueensland,Aust. N Z J Public Health 1996;20:359-363. 29.W ood-D auphinee SW, G authier L, G andek B, Magnan L, Pierre U. Readying a US measure of health status, for use in $C$ anada. $C$ lin Invest Med 1997,20: 224-238. 